\title{
Packing of maximal independent mixed arborescences
}

\author{
Hui Gao \\ Center for Discrete Mathematics, \\ Fuzhou University, \\ Fuzhou, Fujian 350108, China \\ gaoh1118@yeah.net
}

\author{
Daqing Yang* \\ Department of Mathematics, \\ Zhejiang Normal University, \\ Jinhua, Zhejiang 321004, China \\ dyang@zjnu.edu.cn
}

March 10, 2020

\begin{abstract}
Király in [On maximal independent arborescence packing, SIAM J. Discrete. Math. 30 (4) (2016), 2107-2114] solved the following packing problem: Given a digraph $D=(V, A)$, a matroid $M$ on a set $S=\left\{s_{1}, \ldots, s_{k}\right\}$ along with a map $\pi: S \rightarrow V$, find $k$ arc-disjoint maximal arborescences $T_{1}, \ldots, T_{k}$ with roots $\pi\left(s_{1}\right), \ldots, \pi\left(s_{k}\right)$, such that, for any $v \in V$, the set $\left\{s_{i}: v \in V\left(T_{i}\right)\right\}$ is independent and its rank reaches the theoretical maximum. In this paper, we give a new characterization for packing of maximal independent mixed arborescences under matroid constraints. This new characterization is simplified to the form of finding a supermodular function that should be covered by an orientation of each strong component of a matroid-based rooted mixed graph. Our proofs come along with a polynomial-time algorithm. Note that our new characterization extends Király's result to mixed graphs, this answers a question that has already attracted some attentions.
\end{abstract}

Keywords: Packing; Arborescence; Mixed graph; Matroid; Supermodularity AMS subject classifications. 05C70, 05C40, 05B35

\section{Introduction}

In this paper, we consider graphs which may have multiple edges or (and) arcs but not loops. Let $D=(V, A)$ be a digraph. A subdigraph $T$ (it may not be spanning) of $D$ is called an $r$-arborescence if its underlying graph is a tree and for any $u \in V(T)$, there is exactly one directed path in $T$ from $r$ to $u$. The vertex $r$ is called root of the arborescence $T$. Edmonds' arborescence packing theorem [8] characterizes directed graphs that contain $k$ arc-disjoint spanning arborescences in terms of a cut condition.

\footnotetext{
*Corresponding author, grant number NSFC 11871439.
} 
Theorem 1.1 ([8]) In a digraph $D=(V, A)$, let $R=\left\{r_{1}, \ldots, r_{k}\right\} \subseteq V$ be a multiset. There exist arc-disjoint spanning $r_{i}$-arborescneces $(i=1, \ldots, k)$ in $D$ if and only if for any $\emptyset \neq X \subseteq V$,

$$
d_{A}^{-}(X) \geq\left|\left\{r_{i}: r_{i} \notin X\right\}\right| .
$$

A mixed graph $F=(V ; E, A)$ is a graph consisting of the set $E$ of undirected edges and the set $A$ of directed arcs. By regarding each undirected edge as a directed arc in both directions, each concept in directed graphs can be naturally extended for mixed graphs. Especially, a subdigraph $P$ of $F$ is a mixed path if its underlying graph is a path and one end of $P$ can be reached from the other. A subdigraph $T$ (it may not be spanning) of $F$ is called an $r$-mixed arborescence if its underlying graph is a tree and for any $u \in V(T)$, there is exactly one mixed path in $T$ from $r$ to $u$. Equivalently, a subgraph $T$ of $F$ is an $r$-mixed arborescence if there exists an orientation of the undirected edges of $T$ such that the obtained subgraph (whose arc set is the union of original arc set and oriented arc set of $T$ ) is an $r$-arborescence.

Let $X_{1}, \ldots, X_{t}$ be disjoint subsets of $V$; we call $\mathcal{P}=\left\{X_{1}, \ldots, X_{t}\right\}$ a subpartition (of $V$ ) and particularly a partition of $V$ if $V=\cup_{j=1}^{t} X_{j}$. For a subpartition $\mathcal{P}$ of $V$, denote $e_{E}(\mathcal{P})=\mid\left\{e \in E: e\right.$ connects distinct $X_{i}$ s in $\mathcal{P}$ or connects some $X_{i}$ and $\left.V \backslash \cup_{j=1}^{t} X_{j}\right\} \mid$.

For nonempty $X, Z \subseteq V$, let $E(X, Z)$ and $A(X, Z)$ denote the set of edges with one endvertex in $X$ and the other in $Z$ and the set of arcs from $X$ to $Z$ respectively. For simplicity, denote $E(X)=E(X, X)$ and $A(X)=A(X, X)$. Let $Z \rightarrow X$ denote that $X$ and $Z$ are disjoint and $X$ is reachable from $Z$, that is, there is a mixed path from $Z$ to $X$. We shall write $v$ for $\{v\}$ for simplicity. Let $W(X):=X \cup\{v \in V \backslash X: v \rightarrow X\}$.

Let $R=\left\{r_{1}, \ldots, r_{k}\right\} \subseteq V$ be a specified multiset. Let $U_{i}$ be the set of vertices reachable from $r_{i}$. For $u, v \in V$, we say $u \sim v$ if $\left\{i: u \in U_{i}\right\}=\left\{i: v \in U_{i}\right\}$; this $\sim$ is an equivalent relation. Denote equivalent classes for $\sim$ by $\Gamma_{1}, \ldots, \Gamma_{t}$, and we call each $\Gamma_{j}$ an atom. An $r_{i}$-mixed arborescence $T_{i}$ is said to be maximal if $V\left(T_{i}\right)=U_{i}$ (i.e. it spans all the vertices that are reachable from $r_{i}$ in $F$ ). A packing of maximal mixed arborescences w.r.t. $R=\left\{r_{1}, \ldots, r_{k}\right\}$ is a collection $\left\{T_{1}, \ldots, T_{k}\right\}$ of mutually edge and arc-disjoint mixed arborescences such that $T_{i}$ has root $r_{i}$ and $V\left(T_{i}\right)=U_{i}$.

The following remarkable extension of Edmonds' theorem (by Kamiyama, Katoh and Takizawa [13]) enables us to find a packing of maximal arborescences $\left\{T_{1}, \ldots, T_{k}\right\}$ w.r.t. $R$ in a digraph (that is $E=\emptyset$ ).

Theorem 1.2 ([13] ) In a digraph $D=(V, A)$, let $R=\left\{r_{1}, \ldots, r_{k}\right\} \subseteq V$ be a multiset. There are arc-disjoint maximal $r_{i}$-arborescences in $D$ for $i=1, \ldots, k$ if and only if for any $\emptyset \neq X \subseteq V$,

$$
d_{A}^{-}(X) \geq\left|\left\{r_{i}: r_{i} \in W(X) \backslash X\right\}\right|
$$

A bi-set $Y=\left\{Y_{O}, Y_{I}\right\}$ is a pair of sets satisfying $Y_{I} \subseteq Y_{O} \subseteq V$. For bi-set $Y$, define $d_{A}^{-}(Y)=\left|\left\{u v \in A: u \in V \backslash Y_{O}, v \in Y_{I}\right\}\right|$. The application of bi-sets for arborescence packings was first studied by Bérczi and Frank [1, 2], see also [6]. Theorem 1.2 was recently studied again by Király, Szigeti and Tanigawa [16] by using a bi-set function; Matsuoka and Tanigawa [17] extended it to mixed graphs. 
Theorem 1.3 ( 17$)$ Let $F=(V ; E, A)$ be a mixed graph, and $r_{1}, \ldots, r_{k} \in V$. Let $U_{i} \subseteq V(i=1, \ldots, k)$ be the set of vertices reachable from $r_{i}$ in $F$. Then, there exists a packing of $r_{i}$-mixed arborescences $(i=1, \ldots, k)$ spanning $U_{i}$ in $F$ if and only if

$$
e_{E}(\mathcal{P})+\sum_{q=1}^{t} d_{A}^{-}\left(X^{q}\right) \geq \sum_{q=1}^{t}\left|\left\{r_{i}:\left(X^{q}\right)_{I} \subseteq U_{i} \backslash\left\{r_{i}\right\},\left(\left(X^{q}\right)_{O} \backslash\left(X^{q}\right)_{I}\right) \cap U_{i}=\emptyset\right\}\right|
$$

holds for every family of bi-sets $\left\{X^{1}, \ldots, X^{t}\right\}$ such that $\mathcal{P}=\left\{\left(X^{1}\right)_{I}, \ldots,\left(X^{t}\right)_{I}\right\}$ is a subpartition of some atom $\Gamma_{j}$ and that $\left(\left(X^{q}\right)_{O} \backslash\left(X^{q}\right)_{I}\right) \cap \Gamma_{j}=\emptyset$ holds for $q=1, \ldots, t$.

Let $M$ be a matroid on a set $S$ with rank function $r_{M}$, and $\pi: S \rightarrow V$ be a (not necessarily injective) map. We may think of $\pi$ as a placement of the elements of $S$ at vertices of $V$ and different elements of $S$ may be placed at the same vertex. For related definitions and properties of matroids, we refer to [10]. We say that the quadruple $(F, M, S, \pi)$ is a matroid-based rooted mixed graph (or a matroid-based rooted digraph if $E=\emptyset)$.

The following definition was introduced by Katoh and Tanigawa [14. $\pi$ is called $M$-independent if $\pi^{-1}(v)$ is independent in $M$ for each $v \in V$. For $X \subseteq V$, denote by $S_{X}$ the set $\pi^{-1}(X)$. An $M$-based packing of mixed arborescences is a set $\left\{T_{1}, \ldots, T_{|S|}\right\}$ of pairwise edge and arc-disjoint mixed arborescences for which $T_{i}$ has root $\pi\left(s_{i}\right)$ for $i=1, \ldots,|S|$ (where $S=\left\{s_{1}, \ldots, s_{t}\right\}$ ), and for each $v \in V$, the set $\left\{s_{j} \in S: v \in V\left(T_{j}\right)\right\}$ is a base of $S$ (we also say that $s_{i}$ is the root of $T_{i}$ ). Durand de Gevigney, Nguyen and Szigeti [7] involve Edmonds' Theorem with the above packing version.

Theorem 1.4 ([7]) Let $(D, M, S, \pi)$ be a matroid-based rooted digraph. There exists an $M$-based packing of arborescences in $(D, M, S, \pi)$ if and only if $\pi$ is $M$-independent and

$$
d_{D}^{-}(X) \geq r_{M}(S)-r_{M}\left(S_{X}\right)
$$

holds for every $\emptyset \neq X \subseteq V$.

A maximal $M$-independent packing of mixed arborescences is a set $\left\{T_{1}, \ldots, T_{|S|}\right\}$ of pairwise edge and arc-disjoint mixed arborescences for which $T_{i}$ has root $\pi\left(s_{i}\right)$ for $i=1, \ldots,|S|$, the set $\left\{s_{i} \in S: v \in V\left(T_{i}\right)\right\}$ is independent in $M$, and $\mid\left\{s_{i} \in S: v \in\right.$ $\left.V\left(T_{i}\right)\right\} \mid=r_{M}\left(S_{W(v)}\right)$ for each $v \in V$. And Király [15] characterized a digraph containing such a packing, extending both Theorem 1.2 and Theorem 1.4.

Theorem 1.5 ([15]) Let $(D=(V, A), M, S, \pi)$ be a matroid-based rooted digraph. There exists a maximal $M$-independent packing of arborescences in $(D, M, S, \pi)$ if and only if $\pi$ is $M$-independent and

$$
d_{A}^{-}(X) \geq r_{M}\left(S_{W(X)}\right)-r_{M}\left(S_{X}\right)
$$

holds for each $\emptyset \neq X \subseteq V$. 
Fortier, Király, Léonard, Szigeti and Talon [9] had mentioned the following research problem: how to extend Theorem 1.5 to mixed graphs (therefore also generalize Theorem 1.3 to allow matroid constraints). Matsuoka and Tanigawa [17] remarked that Theorem 1.3 can be established in a more general setting by allowing matroid constraints. This way of generalization relies on some recent results on the reachability arborescence packing by Király, Szigeti and Tanigawa [16].

In this paper, we give a new characterization for packing of maximal independent mixed arborescences under matroid constraints. This new characterization is simplified to the form of finding an intersecting supermodular function that should be covered (to be defined at the beginning of Section 2) by an orientation of each strong component of a matroid-based rooted mixed graph $F$. Recall that $C$ is a strong component of $F$ if it is a maximal subgraph of $F$ for which for any two vertices $u, v$ of $C, u$ and $v$ are reachable from each other in $C$. Our new characterization (main result) is the following theorem, and the simplified form is Statement (iii). Note that our new characterization extends Theorem 1.5 to mixed graphs.

Theorem 1.6 Let $(F=(V ; E, A), M, S, \pi)$ be a matroid-based rooted mixed graph. Then the following statements are equivalent.

(i) $\exists$ a maximal $M$-independent packing of mixed arborescences in $(F, M, S, \pi)$.

(ii) $\pi$ is $M$-independent; and

$$
e_{E}(\mathcal{P})+\sum_{q=1}^{t} d_{A}^{-}\left(X^{q}\right) \geq \sum_{q=1}^{t}\left(r_{M}\left(S_{W(V(C))}\right)-r_{M}\left(S_{\left(X^{q}\right)_{O}}\right)\right)
$$

holds for any family of bi-sets $\left\{X^{1}, \ldots, X^{t}\right\}$ such that $\mathcal{P}=\left\{\left(X^{1}\right)_{I}, \ldots,\left(X^{t}\right)_{I}\right\}$ is a vertex subpartition of some strong component $C$ and $\left(X^{q}\right)_{O} \backslash\left(X^{q}\right)_{I}=W(Y)$ for some $Y \subseteq W(V(C)) \backslash V(C)$, where $q=1, \ldots, t$.

(iii) $\pi$ is $M$-independent; and

$$
e_{E}(\mathcal{P}) \geq \sum_{q=1}^{t} f_{C}\left(X_{q}\right)
$$

holds for any strong component $C$ of $F$ and subpartition $\mathcal{P}=\left\{X_{1}, \ldots, X_{t}\right\}$ of $V(C)$, where $f_{C}\left(X_{q}\right)=\max \left\{r_{M}\left(S_{W(V(C))}\right)-r_{M}\left(S_{X}\right)-d_{A}^{-}(X): X_{q} \subseteq X\right.$ and $X \backslash X_{q}=W(Y)$ for some $\left.Y \subseteq W(V(C)) \backslash V(C)\right\}$.

The proof of the main result is Section 2. Here we show that Theorem $\mathbf{1 . 6}$ deduces the sufficiency of Theorem 1.3. Thus it generalizes Theorem 1.3 to allow matroid constraints, therefore this new characterization provides a new and simple solution to the above mentioned research problem.

Let $r_{1}, \ldots, r_{k} \in V, S=\{1, \ldots, k\}$ and $\pi: S \rightarrow V$ such that $\pi(i)=r_{i}$ for $i=1, \ldots, k$. Let $M$ be a free matroid on $S$. Then a maximal $M$-independent packing of mixed 
arborescences is exactly a packing of maximal mixed arborescences w.r.t. $\left\{r_{1}, \ldots, r_{k}\right\}$. Then it suffices to show the following fact:

Fact 1.7 If (1) holds, then (3) holds.

Proof. Suppose (11) holds. Let $C$ be a strong component of $F$ and $X=\left(X_{O}, X_{I}\right)$ a bi-set such that $X_{I} \subseteq V(C)$ and $X_{O} \backslash X_{I}=W(Y)$ for some $Y \subseteq W(V(C)) \backslash V(C)$. Note that $d_{A}^{-}(X)=d_{A}^{-}\left(X_{O}\right)-\left|A\left(V \backslash X_{O}, W(Y)\right)\right|$ and $A\left(V \backslash X_{O}, W(Y)\right)=\emptyset$, thus

$$
d_{A}^{-}(X)=d_{A}^{-}\left(X_{O}\right)
$$

Let $s \in S$ and $U_{s}$ be the set of vertices reachable from $\pi(s)$ in $F$. Then $\pi(s) \in W(V(C))$ if and only if $X_{I} \subseteq U_{s}$; and $\pi(s) \notin W(Y)$ if and only if $W(Y) \cap U_{s}=\emptyset$. Since $M$ is a free matroid and $X_{O}=X_{I} \cup W(Y)$, we have

$$
\begin{aligned}
& r_{M}\left(S_{W(V(C))}\right)-r_{M}\left(S_{X_{O}}\right)=\left|S_{W(V(C))-X_{I}-W(Y)}\right| \\
& =\mid\left\{s \in S: \pi(s) \in W(V(C)), \pi(s) \notin X_{I}, \text { and } \pi(s) \notin W(Y)\right\} \mid \\
& =\mid\left\{s \in S: X_{I} \subseteq U_{s}, \pi(s) \notin X_{I}, \text { and } W(Y) \cap U_{s}=\emptyset\right\} \mid .
\end{aligned}
$$

For any two $u, v \in V(C), u \sim v$ (by definition); thus $V(C) \subseteq \Gamma_{j}$ for some atom $\Gamma_{j}$.

If $W(Y) \cap \Gamma_{j} \neq \emptyset$, then $\pi(s) \in W(V(C))$ implies $\pi(s) \in W(Y)$; thus $S_{W(V(C))} \subseteq$ $S_{W(Y)} \subseteq S_{X_{O}}$ and

$$
r_{M}\left(S_{W(V(C))}\right)-r_{M}\left(S_{X_{O}}\right)-d_{A}^{-}\left(X_{O}\right) \leq 0
$$

Let $\left\{X^{1}, \ldots, X^{t}\right\}$ be a family of bi-sets such that $\mathcal{P}=\left\{\left(X^{1}\right)_{I}, \ldots,\left(X^{t}\right)_{I}\right\}$ is a subpartition of $V(C)$ and that $\left(X^{q}\right)_{O} \backslash\left(X^{q}\right)_{I}=W(Y)$ for some $Y \subseteq W(V(C)) \backslash V(C)$, where $q=1, \ldots, t$. Then we have

$$
\begin{aligned}
& e_{E}(\mathcal{P}) \geq e_{E}\left(\left\{\left(X^{q}\right)_{I}:\left(\left(X^{q}\right)_{O} \backslash\left(X^{q}\right)_{I}\right) \cap \Gamma_{j}=\emptyset\right\}\right) \\
& \geq \sum_{\left(\left(X^{q}\right)_{O} \backslash\left(X^{q}\right)_{I}\right) \cap \Gamma_{j}=\emptyset}\left(\left|\left\{s:\left(X^{q}\right)_{I} \subseteq U_{s} \backslash\{\pi(s)\},\left(\left(X^{q}\right)_{O} \backslash\left(X^{q}\right)_{I}\right) \cap U_{s}=\emptyset\right\}\right|-d_{A}^{-}\left(X^{q}\right)\right) \\
& \text { (by (11)) } \\
& =\sum_{\left(\left(X^{q}\right)_{O} \backslash\left(X^{q}\right)_{I}\right) \cap \Gamma_{j}=\emptyset}\left(r_{M}\left(S_{W(V(C))}\right)-r_{M}\left(S_{\left(X^{q}\right)_{O}}\right)-d_{A}^{-}\left(\left(X^{q}\right)_{O}\right) \quad \text { (by (15) and (6) }\right) \\
& \left.\geq \sum_{q=1}^{t}\left(r_{M}\left(S_{W(V(C))}\right)-r_{M}\left(S_{\left(X^{q}\right)_{O}}\right)-d_{A}^{-}\left(\left(X^{q}\right)_{O}\right)\right) \quad \text { (by (77) }\right) \text {. }
\end{aligned}
$$

That is, (3) holds. 


\section{Proof of Theorem 1.6}

Let $\Omega$ be a set and $X_{1}, X_{2} \subseteq \Omega . X_{1}$ and $X_{2}$ are intersecting if $X_{1} \cap X_{2} \neq \emptyset$. A function $p: 2^{\Omega} \rightarrow \mathbb{Z}$ is supermodular (intersecting supermodular) if the inequality

$$
p(X)+p(Y) \leq p(X \cup Y)+p(X \cap Y)
$$

holds for all subsets (intersecting subsets, respectively) of $\Omega$. A function $b$ is submodular if $-b$ is supermodular. For some recent work related to supermodularity in graph optimization, refer to [3, 4, 5, 12,

A family $\mathcal{H}$ of subsets of $V$ is intersecting if for any $X, Y \in \mathcal{H}, X \cap Y \in \mathcal{H}$. For a set function $f: \mathcal{H} \rightarrow \mathbb{Z}$, a directed graph $D=(V, A)$ (or just $A$ ) is said to cover $f$ if $d_{A}^{-}(X) \geq f(X)$ holds for all $X \in \mathcal{H}$.

\subsection{Preliminaries}

Let $(D=(V, A), M, S, \pi)$ be a matroid-based rooted digraph. Suppose (2) holds for each $\emptyset \neq X \subseteq V$, we say $X_{0} \subseteq V$ is tight if the equality of (2) holds. Note that the in-degree function $d_{A}^{-}$of $D$ and rank function of a matroid is submodular.

Lemma 2.1 (Lemma 10 of [15], adapted) Let $(D=(V, A), M, S, \pi)$ be a matroid-based rooted digraph for which (2) holds for each $\emptyset \neq X \subseteq V$. Let $u v \in A$ and $X_{0}$ be a minimal tight set such that the arc uv enters $X_{0}$. Then $X_{0} \subseteq W(v)$.

Lemma 2.2 Let $(D=(V, A), M, S, \pi)$ be a matroid-based rooted digraph. There exists a maximal $M$-independent packing of arborescences in $(D, M, S, \pi)$ if and only if $\pi$ is $M$-independent and (2) holds for $X \subseteq V$ such that $v \in X \subseteq W(v)$ for some $v \in V$.

Proof. The necessity comes from Theorem 1.5 directly.

For the sufficiency, suppose to the contrary that $D$ does not have such a packing. By Theorem 1.5, there exists $X_{0} \subseteq V$ such that $d_{A}^{-}\left(X_{0}\right)<r_{M}\left(S_{W\left(X_{0}\right)}\right)-r_{M}\left(S_{X_{0}}\right)$. Let $D^{\prime}=\left(V, A^{\prime}\right)$ be a minimal digraph for which: $(i) A \subseteq A^{\prime},(i i) W_{D}(v)=W_{D^{\prime}}(v)$ for each $v \in V$, and $(i i i) d_{A^{\prime}}^{-}(X) \geq r_{M}\left(S_{W(X)}\right)-r_{M}\left(S_{X}\right)$ for $X \subseteq V$. Note that such a digraph exists because we can always add arcs $u v$ with $u \in W_{D}(v)$ till Condition (iii) holds. Then $d_{A^{\prime}}^{-}\left(X_{0}\right) \geq r_{M}\left(S_{W\left(X_{0}\right)}\right)-r_{M}\left(S_{X_{0}}\right)>d_{A}^{-}\left(X_{0}\right)$; and there exists an arc $u_{0} v_{0} \in A^{\prime} \backslash A$.

By the minimality of $D^{\prime}$, there exists $X_{1} \subseteq V$ such that $d_{A^{\prime}-u_{0} v_{0}}^{-}\left(X_{1}\right)<r_{M}\left(S_{W\left(X_{1}\right)}\right)-$ $r_{M}\left(S_{X_{1}}\right)$. Since $d_{A^{\prime}}^{-}\left(X_{1}\right) \geq r_{M}\left(S_{W\left(X_{1}\right)}\right)-r_{M}\left(S_{X_{1}}\right)$, we have $d_{A^{\prime}}^{-}\left(X_{1}\right)=r_{M}\left(S_{W\left(X_{1}\right)}\right)-$ $r_{M}\left(S_{X_{1}}\right)$ (that is $X_{1}$ is tight) and $u_{0} v_{0}$ enters $X_{1}$. Let $X_{2}$ be a minimal tight set of $D^{\prime}$ such that $u_{0} v_{0}$ enters $X_{2}$. Then

$$
d_{A}^{-}\left(X_{2}\right) \leq d_{A^{\prime}-u_{0} v_{0}}^{-}\left(X_{2}\right)<d_{A^{\prime}}^{-}\left(X_{2}\right)=r_{M}\left(S_{W\left(X_{2}\right)}\right)-r_{M}\left(S_{X_{2}}\right) .
$$

But by Lemma 2.1, $v_{0} \in X_{2} \subseteq W\left(v_{0}\right)$. Then by the assumption of this lemma, $d_{A}^{-}\left(X_{2}\right) \geq r_{M}\left(S_{W\left(X_{2}\right)}\right)-r_{M}\left(S_{X_{2}}\right)$, a contradiction to (8)). 
Theorem 2.3 ([11]) Let $G=(V, E)$ be an undirected graph, $\mathcal{H} \subseteq 2^{V}$ be an intersecting family with $\emptyset \notin \mathcal{H}$ and $V \in \mathcal{H}$, and $f: \mathcal{H} \rightarrow R$ an intersecting supermodular function with $f(V)=0$. There exists an orientation of $E$ that covers $f$ (that is $d_{A}^{-}(X) \geq f(X)$ for all $X \in \mathcal{H}$, where $A$ is the oriented arc set of $E$ ) if and only if

$$
e_{E}(\mathcal{P}) \geq \sum_{i=1}^{t} f\left(V_{i}\right)
$$

holds for every collection $\mathcal{P}=\left\{V_{1}, \ldots, V_{t}\right\}$ of mutually disjoint members of $\mathcal{H}$.

\subsection{Proof of Theorem 1.6}

We shall show that $(i) \Rightarrow(i i),(i i) \Rightarrow(i i i)$, and $(i i i) \Rightarrow(i)$, this will finish the proof. (i) $\Rightarrow$ (ii): Suppose there exists a maximal $M$-independent packing of mixed arborescences in $(F, M, S, \pi)$, then there exists an orientation $A^{\prime}$ of $E$ such that in $\left(D^{\prime}=\right.$ $\left.\left(V, A \cup A^{\prime}\right), M, S, \pi\right)$ there exists a maximal $M$-independent packing of arborescences. By Theorem 1.5,

$$
\begin{aligned}
& d_{A \cup A^{\prime}}^{-}\left(\left(X^{q}\right)_{O}\right) \geq r_{M}\left(S_{W\left(\left(X^{q}\right)_{O}\right)}\right)-r_{M}\left(S_{\left(X^{q}\right)_{O}}\right), \text { and then } \\
& d_{A^{\prime}}^{-}\left(\left(X^{q}\right)_{O}\right) \geq r_{M}\left(S_{W\left(\left(X^{q}\right)_{O}\right)}\right)-r_{M}\left(S_{\left(X^{q}\right)_{O}}\right)-d_{A}^{-}\left(\left(X^{q}\right)_{O}\right) .
\end{aligned}
$$

By the definition of $d_{A^{\prime}}^{-}\left(X^{q}\right)$ for bi-set $X^{q}$, we have $d_{A^{\prime}}^{-}\left(\left(X^{q}\right)_{O}\right)=d_{A^{\prime}}^{-}\left(X^{q}\right)+\mid A^{\prime}(V \backslash$ $\left.\left(X^{q}\right)_{O}, W(Y)\right) \mid$, and $d_{A^{\prime}}^{-}\left(\left(X^{q}\right)_{I}\right)=d_{A^{\prime}}^{-}\left(X^{q}\right)+\left|A^{\prime}\left(W(Y),\left(X^{q}\right)_{I}\right)\right|$. Similarly, $d_{A}^{-}\left(\left(X^{q}\right)_{O}\right)=$ $d_{A}^{-}\left(X^{q}\right)+\left|A\left(V \backslash\left(X^{q}\right)_{O}, W(Y)\right)\right|$.

Since $\left(X^{q}\right)_{O}=W(Y) \cup\left(X^{q}\right)_{I}$ for some $Y \subseteq W(V(C)) \backslash V(C)$, and $\left(X^{q}\right)_{I} \subseteq V(C)$, we have $W\left(\left(X^{q}\right)_{O}\right)=W(V(C)), A^{\prime}\left(V \backslash\left(X^{q}\right)_{O}, W(Y)\right)=\emptyset$, and $A\left(V \backslash\left(X^{q}\right)_{O}, W(Y)\right)=\emptyset$.

Since $C$ is a strong component, there is no edge in $E$ between $V(C)$ and $V \backslash V(C)$; since $\left(X^{q}\right)_{I} \subseteq V(C)$, and $Y \subseteq W(V(C)) \backslash V(C)$ (then $W(Y) \subseteq W(V(C)) \backslash V(C)$ ), we have $A^{\prime}\left(W(Y),\left(X^{q}\right)_{I}\right)=\emptyset$.

Hence,

$$
d_{A^{\prime}}^{-}\left(\left(X^{q}\right)_{O}\right)=d_{A^{\prime}}^{-}\left(\left(X^{q}\right)_{I}\right)=d_{A^{\prime}}^{-}\left(X^{q}\right), \quad d_{A}^{-}\left(\left(X^{q}\right)_{O}\right)=d_{A}^{-}\left(X^{q}\right) .
$$

It follows that,

$$
\begin{aligned}
& e_{E}(\mathcal{P}) \geq \sum_{q=1}^{t} d_{A^{\prime}}^{-}\left(\left(X^{q}\right)_{I}\right) \quad\left(\text { since } A^{\prime} \text { is an orientation of } E\right) \\
& =\sum_{q=1}^{t} d_{A^{\prime}}^{-}\left(\left(X^{q}\right)_{O}\right) \quad(\text { by (100) }) \\
& \geq \sum_{q=1}^{t}\left(r_{M}\left(S_{W\left(\left(X^{q}\right)_{O}\right)}\right)-r_{M}\left(S_{\left(X^{q}\right)_{O}}\right)-d_{A}^{-}\left(\left(X^{q}\right)_{O}\right)\right) \quad(\text { by (9) }) \\
& =\sum_{q=1}^{t}\left(r_{M}\left(S_{W(V(C))}\right)-r_{M}\left(S_{\left(X^{q}\right)_{O}}\right)-d_{A}^{-}\left(X^{q}\right)\right) \quad\left(\text { by } W\left(\left(X^{q}\right)_{O}\right)=W(V(C)) \text { and (10) }\right) .
\end{aligned}
$$


(ii) $\Rightarrow$ (iii): For $1 \leq q \leq t$, suppose $Y_{q}$ satisfies that $f_{C}\left(X_{q}\right)=r_{M}\left(S_{W(V(C))}\right)-r_{M}\left(S_{Y_{q}}\right)-$ $d_{A}^{-}\left(Y_{q}\right)$, and $X_{q} \subseteq Y_{q}$ and $Y_{q} \backslash X_{q}=W(Y)$ for some $Y \subseteq W(V(C)) \backslash V(C)$; define bi-set $X^{q}=\left(Y_{q}, X_{q}\right)$. Since $(i i)$ holds, we have

$$
\begin{array}{rlrl}
e_{E}(\mathcal{P})+\sum_{q=1}^{t} d_{A}^{-}\left(Y_{q}\right) & =e_{E}(\mathcal{P})+\sum_{q=1}^{t} d_{A}^{-}\left(\left(X^{q}\right)_{O}\right)=e_{E}(\mathcal{P})+\sum_{q=1}^{t} d_{A}^{-}\left(X^{q}\right) & & \text { (by (10) }) \\
& \geq \sum_{q=1}^{t}\left(r_{M}\left(S_{P(V(C))}\right)-r_{M}\left(S_{\left(X^{q}\right)_{O}}\right)\right) & \text { (by (3id) }) \\
& =\sum_{q=1}^{t}\left(r_{M}\left(S_{P(V(C))}\right)-r_{M}\left(S_{Y_{q}}\right)\right) &
\end{array}
$$

that is,

$$
e_{E}(\mathcal{P}) \geq \sum_{q=1}^{t}\left(r_{M}\left(S_{P(V(C))}\right)-r_{M}\left(S_{Y_{q}}\right)-d_{A}^{-}\left(Y_{q}\right)\right)=\sum_{q=1}^{t} f_{C}\left(X_{q}\right) .
$$

(iii) $\Rightarrow$ (i): Let $\tau(F)$ be the the number of strong components of $F$. We prove that $($ iii $) \Rightarrow(i)$ by induction on $\tau(F)$.

For the base step, suppose $\tau(F)=1$, i.e., $F$ is strongly connected. Then, for any subpartition $\left\{X_{1}, \ldots, X_{t}\right\}$ of $V(F)$, by (4), we have

$$
e_{E}(\mathcal{P}) \geq \sum_{q=1}^{t} f_{F}\left(X_{q}\right)=\sum_{q=1}^{t}\left(r_{M}(S)-r_{M}\left(S_{X_{q}}\right)-d_{A}^{-}\left(X_{q}\right)\right) .
$$

Since $r_{M}$ and $d_{A}^{-}$are submodular, we have $r_{M}(S)-r_{M}\left(S_{X}\right)-d_{A}^{-}(X)$ is intersecting supermodular on $2^{V}$, and $f(V)=0$.

By Theorem 2.3, there exists an orientation $A_{0}$ of $E$ such that the digraph $D_{0}=$ $\left(V, A_{0}\right)$ covers $r_{M}(S)-r_{M}\left(S_{X}\right)-d_{A}^{-}(X)$, i.e., $d_{A_{0}}^{-}(X) \geq r_{M}(S)-r_{M}\left(S_{X}\right)-d_{A}^{-}(X)$; this is the same as $D_{0}^{\prime}=\left(V, A \cup A_{0}\right)$ covers $r_{M}(S)-r_{M}\left(S_{X}\right)$. By Theorem 1.4, there exists an $M$-based packing of arborescences in $\left(D_{0}^{\prime}, M, S, \pi\right)$.

For the induction step, suppose $\tau(F)=n \geq 2$, and suppose that $($ iii $) \Rightarrow(i)$ holds for $\tau(F) \leq n-1$.

First we show that there exists a strong component $C_{0}$ of $F$ such that no arcs come out of $C_{0}$. Assume otherwise, then each strong component has arcs coming out of it. But then $F$ itself is strongly connected, a contradiction to $\tau(F) \geq 2$. Suppose $C_{0}$ is such a strong component, $F_{1}$ is the induced mixed graph on vertex set $V\left(F_{1}\right):=V(F) \backslash V\left(C_{0}\right)$. Then $\tau\left(F_{1}\right)=n-1$.

The following fact is heavily used: $E\left(V\left(C_{0}\right), V\left(F_{1}\right)\right)=\emptyset, A\left(V\left(C_{0}\right), V\left(F_{1}\right)\right)=\emptyset$; therefore for $X_{0} \subseteq V\left(F_{1}\right), W_{F_{1}}\left(X_{0}\right)=W\left(X_{0}\right) \subseteq V\left(F_{1}\right)$.

By the induction hypothesis, there exists a maximal $M \mid S_{V\left(F_{1}\right)}$-independent packing of mixed arborescences in $F_{1}$; that is, there exist pairwise arc disjoint mixed $\pi\left(s_{i}\right)$ arborescences $T_{i}^{\prime}$ in $F_{1}$, where $1 \leq i \leq\left|S_{V\left(F_{1}\right)}\right|$; and for any $v \in V\left(F_{1}\right),\left\{s_{i}: v \in\right.$ 
$\left.V\left(T_{i}^{\prime}\right)\right\}$ is independent and $\left|\left\{s_{i}: v \in V\left(T_{i}^{\prime}\right)\right\}\right|=r_{M}\left(S_{W(v)}\right)$. Equivalently, $E\left(F_{1}\right)$ can be oriented to $A_{1}$ such that there exist pairwise arc disjoint $\pi\left(s_{i}\right)$-arborescences $T_{i}$ in $D_{1}:=$ $\left(V\left(F_{1}\right), A\left(V\left(F_{1}\right)\right) \cup A_{1}\right)$, where $1 \leq i \leq\left|S_{V\left(F_{1}\right)}\right|$; and for any $v \in V\left(F_{1}\right),\left\{s_{i}: v \in V\left(T_{i}\right)\right\}$ is independent and $\left|\left\{s_{i}: v \in V\left(T_{i}\right)\right\}\right|=r_{M}\left(S_{W(v)}\right)$.

By Theorem 1.5, for any $\emptyset \neq X_{0} \subseteq V\left(F_{1}\right)$,

$$
d_{D_{1}}^{-}\left(X_{0}\right) \geq r_{M}\left(S_{W_{D_{1}}\left(X_{0}\right)}\right)-r_{M}\left(S_{X_{0}}\right) .
$$

Note that if $v \in V\left(T_{i}\right)$, then $\pi\left(s_{i}\right) \in W_{D_{1}}(v)$. Thus $\left\{s_{i}: v \in V\left(T_{i}\right)\right\} \subseteq S_{W_{D_{1}}(v)}$, and $\left|\left\{s_{i}: v \in V\left(T_{i}\right)\right\}\right| \leq r_{M}\left(S_{W_{D_{1}}(v)}\right)$. Since $W_{D_{1}}(v) \subseteq W(v)$, we have $r_{M}\left(S_{W_{D_{1}}(v)}\right) \leq$ $r_{M}\left(S_{W(v)}\right)$. Since $\left|\left\{s_{i}: v \in V\left(T_{i}\right)\right\}\right|=r_{M}\left(S_{W(v)}\right)$, we have $r_{M}\left(S_{W_{D_{1}}}(v)\right)=r_{M}\left(S_{W(v)}\right)$. Thus $r_{M}\left(S_{W_{D_{1}}\left(X_{0}\right)}\right)=r_{M}\left(S_{W\left(X_{0}\right)}\right)$. And $E\left(V\left(C_{0}\right), V\left(F_{1}\right)\right)=A\left(V\left(C_{0}\right), V\left(F_{1}\right)\right)=\emptyset$ gives that $d_{D_{1}}^{-}\left(X_{0}\right)=d_{A \cup A_{1}}^{-}\left(X_{0}\right)$. So (11) can be transformed to:

$$
d_{A \cup A_{1}}^{-}\left(X_{0}\right) \geq r_{M}\left(S_{W\left(X_{0}\right)}\right)-r_{M}\left(S_{X_{0}}\right) .
$$

Define $f_{C_{0}}: 2^{V\left(C_{0}\right)} \backslash\{\emptyset\} \rightarrow Z, f_{C_{0}}(X)=\max \left\{r_{M}\left(S_{W\left(V\left(C_{0}\right)\right)}\right)-r_{M}\left(S_{X_{0}}\right)-d_{A}^{-}\left(X_{0}\right)\right.$ : $X \subseteq X_{0}$ and $X_{0} \backslash X=W(Y)$ for some $\left.Y \subseteq W\left(V\left(C_{0}\right)\right) \backslash V\left(C_{0}\right)\right\}$. Then we have the following claim.

Claim $2.4 f_{C_{0}}$ is intersecting supermodular.

Proof. Suppose $X_{1}, X_{2} \subseteq V\left(C_{0}\right)$ are intersecting sets, $Y_{1}, Y_{2} \subseteq W\left(V\left(C_{0}\right)\right) \backslash V\left(C_{0}\right)$ such that $f_{C_{0}}\left(X_{i}\right)=r_{M}\left(S_{W\left(V\left(C_{0}\right)\right)}\right)-r_{M}\left(S_{X_{i} \cup W\left(Y_{i}\right)}\right)-d_{A}^{-}\left(X_{i} \cup W\left(Y_{i}\right)\right)$ for some $Y_{i} \subseteq$ $W\left(V\left(C_{0}\right)\right) \backslash V\left(C_{0}\right)$, where $i=1,2$.

Note that $W\left(Y_{1}\right) \cup W\left(Y_{2}\right)=W\left(Y_{1} \cup Y_{2}\right)$. Let $Y_{3}=W\left(Y_{1}\right) \cap W\left(Y_{2}\right)$, note that $W\left(Y_{3}\right)=Y_{3}$; thus $W\left(Y_{1}\right) \cap W\left(Y_{2}\right)=W\left(Y_{3}\right)$. Since $r_{M}$ and $d_{A}^{-}$are submodular,

$$
\begin{aligned}
f_{C_{0}}\left(X_{1}\right)+f_{C_{0}}\left(X_{2}\right)=r_{M}\left(S_{W\left(V\left(C_{0}\right)\right)}\right)-r_{M}\left(S_{X_{1} \cup W\left(Y_{1}\right)}\right)-d_{A}^{-}\left(X_{1} \cup W\left(Y_{1}\right)\right) \\
\quad+r_{M}\left(S_{W\left(V\left(C_{0}\right)\right)}\right)-r_{M}\left(S_{X_{2} \cup W\left(Y_{2}\right)}\right)-d_{A}^{-}\left(X_{2} \cup W\left(Y_{2}\right)\right) \\
\leq r_{M}\left(S_{W\left(V\left(C_{0}\right)\right)}\right)-r_{M}\left(S_{X_{1} \cup X_{2} \cup W\left(Y_{1} \cup Y_{2}\right)}\right)-d_{A}^{-}\left(X_{1} \cup X_{2} \cup W\left(Y_{1} \cup Y_{2}\right)\right) \\
+r_{M}\left(S_{W\left(V\left(C_{0}\right)\right)}\right)-r_{M}\left(S_{\left(X_{1} \cap X_{2}\right) \cup W\left(Y_{3}\right)}\right)-d_{A}^{-}\left(\left(X_{1} \cap X_{2}\right) \cup W\left(Y_{3}\right)\right) \\
\leq f_{C_{0}}\left(X_{1} \cup X_{2}\right)+f_{C_{0}}\left(X_{1} \cap X_{2}\right) .
\end{aligned}
$$

Using Claim 2.4 and (4), by Theorem 2.3, we know that there exists an orientation $A_{0}$ of $E\left(C_{0}\right)$ such that $A_{0}$ covers $f_{C_{0}}$, i.e., for any $\emptyset \neq X \subseteq V\left(C_{0}\right)$ and $X_{0}$ such that $X \subseteq X_{0}$ and $X_{0} \backslash X=W(Y)$ for some $Y \subseteq W\left(V\left(C_{0}\right)\right) \backslash V\left(C_{0}\right)$,

$$
d_{A_{0}}^{-}(X) \geq r_{M}\left(S_{W\left(V\left(C_{0}\right)\right)}\right)-r_{M}\left(S_{X_{0}}\right)-d_{A}^{-}\left(X_{0}\right) .
$$

Similarly to (100), we have $d_{A_{0}}^{-}(X)=d_{A_{0}}^{-}\left(X_{0}\right)$. Then for each $X_{0} \subseteq W\left(V\left(C_{0}\right)\right)$ such that $X:=X_{0} \cap V\left(C_{0}\right) \neq \emptyset$ and $W\left(X_{0} \backslash V\left(C_{0}\right)\right)=X_{0} \backslash V\left(C_{0}\right)$, we have

$$
d_{A \cup A_{0}}^{-}\left(X_{0}\right)=d_{A_{0}}^{-}(X)+d_{A}^{-}\left(X_{0}\right) \geq r_{M}\left(S_{W\left(V\left(C_{0}\right)\right)}\right)-r_{M}\left(S_{X_{0}}\right) .
$$

Using orientation $A_{1}$ of $E\left(F_{1}\right)$ and $A_{0}$ of $E\left(C_{0}\right)$, we have a directed graph $D$ of $F$ with arc set $A \cup A_{0} \cup A_{1}$. 
Lemma 2.5 Suppose $v \in V\left(C_{0}\right)$ and $v \in X_{0} \subseteq W(v)$. Then we have

$$
d_{A \cup A_{0} \cup A_{1}}^{-}\left(X_{0}\right) \geq r_{M}\left(S_{W\left(X_{0}\right)}\right)-r_{M}\left(S_{X_{0}}\right) .
$$

Proof. Since $v \in V\left(C_{0}\right)$, then $X_{0} \subseteq W(v)=W\left(V\left(C_{0}\right)\right)$, and $v \in X_{0} \cap V\left(C_{0}\right) \neq \emptyset$. By (13), it suffices to consider the case where $Y:=X_{0} \backslash V\left(C_{0}\right)$ and $Y \varsubsetneqq W(Y)$.

Since $Y \subseteq V\left(F_{1}\right)$, as noted before, $W(Y) \subseteq V\left(F_{1}\right)$. Then $X_{0} \cap W(Y) \subseteq X_{0} \cap$ $\overline{V\left(C_{0}\right)} \subseteq Y \subseteq X_{0} \cap W(Y)$, this gives $X_{0} \cap W(Y)=Y$. Let $X:=X_{0} \cap V\left(C_{0}\right)$, then $X_{0} \cup W(Y)=X \cup W(Y)$. Combining that $r_{M}$ and $d_{A \cup A_{0} \cup A_{1}}^{-}$are submodular, we have

$$
\begin{aligned}
& \left(r_{M}\left(S_{W\left(X_{0}\right)}\right)-r_{M}\left(S_{X_{0}}\right)-d_{A \cup A_{0} \cup A_{1}}^{-}\left(X_{0}\right)\right) \\
+ & \left(r_{M}\left(S_{W(Y)}\right)-r_{M}\left(S_{W(Y)}\right)-d_{A \cup A_{0} \cup A_{1}}^{-}(W(Y))\right) \\
\leq & \left(r_{M}\left(S_{W\left(X_{0}\right)}\right)-r_{M}\left(S_{X \cup W(Y)}\right)-d_{A \cup A_{0} \cup A_{1}}^{-}(X \cup W(Y))\right) \\
+ & \left(r_{M}\left(S_{W(Y)}\right)-r_{M}\left(S_{Y}\right)-d_{A \cup A_{0} \cup A_{1}}^{-}(Y)\right) .
\end{aligned}
$$

Note that $W\left(X_{0}\right)=W\left(V\left(C_{0}\right)\right)$, apply (13) to $X \cup W(Y)$, we have

$$
r_{M}\left(S_{W\left(X_{0}\right)}\right)-r_{M}\left(S_{X \cup W(Y)}\right)-d_{A \cup A_{0} \cup A_{1}}^{-}(X \cup W(Y)) \leq 0 ;
$$

apply (12) to $Y$, we have: $\quad r_{M}\left(S_{W(Y)}\right)-r_{M}\left(S_{Y}\right)-d_{A \cup A_{0} \cup A_{1}}^{-}(Y) \leq 0$; notice that: $d_{A \cup A_{0} \cup A_{1}}^{-}(W(Y))=0$; thus (15) gives $r_{M}\left(S_{W\left(X_{0}\right)}\right)-r_{M}\left(S_{X_{0}}\right)-d_{A \cup A_{0} \cup A_{1}}^{-}\left(X_{0}\right) \leq 0$, this proves the lemma.

We are ready to show $($ iii $) \Rightarrow(i)$ by applying Lemma 2.2. Suppose $X_{0} \subseteq V(F)$, and for some $v \in V(F), v \in X_{0} \subseteq W(v)$. Note that if $v \in V\left(F_{1}\right)$, then $W(v) \subseteq V\left(F_{1}\right)$. If $X_{0} \subseteq V\left(F_{1}\right)$, then by (12), (2) holds. Else $X_{0} \cap V\left(C_{0}\right) \neq \emptyset$, in this case, if vertices $v \in X_{0} \subseteq W(v)$, then $v \in V\left(C_{0}\right)$; by Lemma 2.5, (2) holds. By Lemma 2.2, there exists a maximal $M$-independent packing of mixed arborescences in $(F=(V ; E, A), M, S, \pi)$, this finishes the proof.

Remarks on the complexity: Frank [11] showed that the problem of covering an intersecting supermodular function by orienting edges can be solved in polynomial time. Hence, we can orient all strong components $C$ of $F$ such that the obtained digraph $D$ covers $f_{C}$ in polynomial time. Then, according to the polynomial-time algorithm given in [15], a maximal $M$-independent packing of mixed arborescences in $F$ can be found in polynomial time.

\section{References}

[1] K. Bérczi, A. Frank, Variations for Lovász' submodular ideas, in: M. Grotschel, G.O.H. Katona (Eds.), Building Bridges Between Mathematics and Computer Science, in: Bolyai Society Series: Mathematical Studies, vol. 19, 2008, 137-164.

[2] K. Bérczi, A. Frank, Packing arborescences, in: S. Iwata (Ed.), RIMS Kokyuroku Bessatsu B23: Combinatorial Optimization and Discrete Algorithms, 2010, 1-31. 
[3] K. Bérczi, A. Frank, Supermodularity in unweighted graph optimization I: Branchings and matchings, Math. Oper. Res. 43(3) (2018), 726-753.

[4] K. Bérczi, A. Frank, Supermodularity in unweighted graph optimization II: Matroidal term rank augmentation, Math. Oper. Res. 43 (3) (2018), 754-762.

[5] K. Bérczi, A. Frank, Supermodularity in unweighted graph optimization III: Highlyconnected digraphs, Math. Oper. Res. 43 (3) (2018), 763-780.

[6] K. Bérczi, T. Király, Y. Kobayashi, Covering intersecting bi-set families under matroid constraints. SIAM J. Discrete Math. 30(3), 2016, 1758-1774.

[7] O. Durand de Gevigney, V.-H. Nguyen, Z. Szigeti, Matroid-based packing of arborescences, SIAM J. Discrete Math., 27, 2013, 567-574.

[8] J. Edmonds, Edge-disjoint branchings, Combinatorial algorithms (Courant Comput. Sci. Sympos. 9, New York Univ., New York, 1972), pp. 91-96. Algorithmics Press, New York, 1973.

[9] Q. Fortier, Cs. Király, M. Léonard, Z. Szigeti, A. Talon, Old and new results on packing arborescences in directed hypergraphs, Discrete Appl. Math. 242 (2018), 26-33.

[10] A. Frank, Connections in Combinatorial Optimization, Oxford University Press, Oxford, 2011.

[11] A. Frank, On disjoint trees and arborescences, in: Algebraic Methods in Graph Theory, in: Colloquia Mathematica Societatis Jnos Bolyai, vol. 25, 1978, pp. 159169.

[12] H. Gao, D. Yang, Packing branchings under cardinality constraints on their root sets, arXiv:1908.10795v2 [math.CO] 9 Feb 2020.

[13] N. Kamiyama, N. Katoh, and A. Takizawa, Arc-disjoint in-trees in directed graphs, Combinatorica, 29 (2009) 197-214.

[14] N. Katoh and S. Tanigawa, Rooted-tree decompositions with matroid constraints and the infinitesimal rigidity of frameworks with boundaries, SIAM J. Discrete Math., 27 (2013), pp. 155-185.

[15] Cs. Király, On maximal independent arborescence packing, SIAM J. Discrete. Math. 30 (4) (2016), 2107-2114.

[16] Cs. Király, Z. Szigeti, S. Tanigawa, Packing of Arborescences with Matroid Constraints Via Matroid Intersection, EGRES Technical Reports, TR-2018-08, Egerváry Research Group, 2018. 
PACKING OF MAXIMAL INDEPENDENT MIXED ARBORESCENCES

[17] T. Matsuoka, S. Tanigawa, On reachability mixed arborescence packing, Discrete Optimization 32 (2019) 1-10. 